ARTICLE

Received 9 Feb 2015 | Accepted 10 Feb 2016 | Published 6 Apr 2016

DOI: $10.1038 /$ ncomms11008

OPEN

\title{
Meta-analysis of gene-environment-wide association scans accounting for education level identifies additional loci for refractive error
}

Qiao Fan et al.\#

Myopia is the most common human eye disorder and it results from complex genetic and environmental causes. The rapidly increasing prevalence of myopia poses a major public health challenge. Here, the CREAM consortium performs a joint meta-analysis to test singlenucleotide polymorphism (SNP) main effects and SNP $\times$ education interaction effects on refractive error in 40,036 adults from 25 studies of European ancestry and 10,315 adults from 9 studies of Asian ancestry. In European ancestry individuals, we identify six novel loci (FAM15OB-ACP1, LINC00340, FBN1, DIS3L-MAP2K1, ARID2-SNAT1 and SLC14A2) associated with refractive error. In Asian populations, three genome-wide significant loci $A R E G, G A B R R 1$ and PDE10A also exhibit strong interactions with education $\left(P<8.5 \times 10^{-5}\right)$, whereas the interactions are less evident in Europeans. The discovery of these loci represents an important advance in understanding how gene and environment interactions contribute to the heterogeneity of myopia. 
$\mathrm{M}$ yopia, or nearsightedness, has rapidly emerged as a global health concern in the last three decades ${ }^{1}$. It is one of the leading causes of visual impairment and is associated with potentially blinding ocular complications including myopic maculopathy, glaucoma, cataract and retinal detachment ${ }^{2}$. Evidence from family and twin studies strongly supports the heritability of myopia ${ }^{3}$. Estimates for the heritability of the quantitative trait refractive error have been reported to be as high as $90 \%$ (ref. 4). On the other hand, the rapid upsurge of myopia in the last few decades in many parts of the world is likely to be a consequence of lifestyle changes such as the increasing educational intensity, in particular in urban East Asia ${ }^{5,6}$, and potentially gene and environment $(\mathrm{G} \times \mathrm{E})$ interactions.

Major attempts undertaken in genome-wide association studies (GWAS) to elucidate the genetic determination of myopia and refractive error have recently led to the discovery of $>30$ distinct susceptibility loci ${ }^{7,8}$. Nevertheless, collectively these genetic variants are estimated to explain $<12 \%$ of phenotypic variance in refractive errror ${ }^{7,8}$. As myopia is a result of the combination of genetic and environmental factors, interplay between genes and environment may account for a substantial proportion of the phenotypic variance. In recent times, we showed interactions between education and genetic risk score of myopia derived from 26 known GWAS single-nucleotide polymorphisms (SNPs) in the Rotterdam Study ${ }^{9}$; the combined effect of genetic predisposition and education on the risk of myopia was substantially greater than anticipated from a simple sum of these two factors. At the gene level, some genes such as SHISA6-DNAH9 have been shown to interact with education level and exhibit strong genetic effects for myopia among Asians with at least higher secondary education ${ }^{10}$. In the current study, we demonstrate that new genetic effects implicated in myopia development could be uncovered by studying interactions between genetic variants and education level.

In the context of the aetiology of refractive errors, education attainment is generally considered a surrogate measure for accumulated near work activity ${ }^{1}$. When viewing near objects, the eye generates extra optical power through the process of accommodation to focus the image on the retinal plane, to maintain clear vision ${ }^{11}$. There is an accommodative lag (less accommodation produced than needed) in many myopes, resulting in a hyperopic defocus on the retina for near work, which has long been proposed to promote eye growth ${ }^{1,12}$, but whether this occurs before or after the onset of myopia in humans is less clear. The retina has a central role in the mechanism linking such visual input with eye growth and refractive development ${ }^{13}$. Several neurotransmitters or molecules have been implicated in this process by animal studies including dopamine, acetylcholine, vasoactive intestinal peptide, GABA ( $\gamma$-aminobutyric acid) and glucagon ${ }^{14,15}$. However, an organized framework for the retinal signalling mechanisms underlying refractive error development under various environmental conditions remains to be elucidated.

Factoring in environmental exposures may enhance power for the detection of genes, especially in circumstances where a genetic locus has a differential effect conditional on specific environment exposures ${ }^{16}$. Gene-environment-wide interaction studies (GEWIS) using a joint meta-analysis (JMA) approach on SNP main effects and $\mathrm{SNP} \times$ environment interactions have recently been described ${ }^{17,18}$. This approach has successfully identified six novel loci associated with fasting insulin and glucose accounting for interactions with body mass index ${ }^{18}$. It also led to the identification of two novel loci for pulmonary function that did not emerge from analyses based on the genetic main effects alone ${ }^{19}$. The welldocumented effects of educational attainment on myopia and refractive error make the proposed interaction an excellent analytical candidate for the GEWIS.
The availability of large-scale GWAS spherical equivalent data sets from the Consortium for Refractive Error And Myopia (CREAM) makes $\mathrm{G} \times \mathrm{E}$ interaction analyses feasible. To identify additional genetic variants for refractive error, we performed GEWIS-based analyses on 40,036 adults of European ancestry from 25 studies and 10,315 adults of Asian ancestry from 9 studies. We identified nine new loci using the JMA approach, where three loci exhibited $\mathrm{G} \times \mathrm{E}$ interaction on refractive error in Asians, including the $\mathrm{GABA}_{\mathrm{C}}$ receptor subunit $\rho 1$ gene GABRR1.

\section{Results}

Educational level and its main effects on spherical equivalent. The baseline characteristics of 50,351 participants from 34 studies in our meta-analysis are shown in Table 1. A total of 40,036 participants were of European descent and 10,315 were of Asian descent; the age of the participants ranged from 20 to 99 years. We grouped individuals into two educational categories: a higher education group that included individuals who completed higher secondary or university education and a lower education group comprising those with lower secondary education or below (see Methods). Among Europeans, the proportions of participants in the higher education group ranged from $16.5 \%$ (FITSA $^{20}$ and OGP Talana ${ }^{21}$ ) to $94.4 \%\left(\right.$ AREDS $^{22}$ ) with an average of $50.7 \%$ (Supplementary Table 1). In Asians, the proportions of individuals in the higher education group ranged from $6.7 \%$ $\left(\right.$ SiMES $\left.^{23}\right)$ to $75.9 \%\left(\right.$ Nagahama $\left.^{24}\right)$ with an average of $30.0 \%$. Across all studies, individuals in the higher education group had a spherical equivalent refractive error that was on average 0.59 dioptres (D) more myopic, or less hyperopic, compared with those in the lower education group $(\beta=-0.59 \mathrm{D} ; 95 \%$ confidence interval $(\mathrm{CI}):-0.64$ to $-0.55 \mathrm{D})$. High education level was associated with a twofold more myopic spherical equivalent in individuals of Asian as compared with European ancestry (Asians: $\beta=-1.09 \mathrm{D}, 95 \% \mathrm{CI}:-1.20$ to $-0.98 \mathrm{D}$; Europeans: $\beta=-0.49$ D, $95 \%$ CI: -0.54 to $-0.44 \mathrm{D}$; Fig. 1). Among Asian studies, we also observed heterogeneity of education effects for refractive error. The education effects on spherical equivalent in Singapore Chinese were significantly larger than that in other Asian studies (Singapore Chinese: $\beta=-1.75 \mathrm{D}, 95 \% \mathrm{CI}:-1.92$ to $-1.58 \mathrm{D}$; other Asian cohorts: $\beta=-0.60 \mathrm{D}, 95 \% \mathrm{CI}:-0.75$ to $-0.46 \mathrm{D})$.

GEWIS in Europeans. After stringent quality control (QC) filtering, $\sim 6$ million SNPs in each study were eligible for the genome-wide JMA test (Supplementary Table 2). The JMA for SNP main effects and SNP $\times$ education interactions in 40,036 European Ancestry individuals showed an association with spherical equivalent at 12 previously implicated loci (Fig. 2a, Supplementary Table 3 and Supplementary Fig 1). We also identified four previously unreported loci associated with spherical equivalent achieving genome-wide significance $\left(P_{\text {IMA }}<5.0 \times 10^{-8} ; \quad P_{\text {het }} \geq 0.086 ;\right.$ Table 2): FAM150B-ACP1, LINC00340, FBN1 and DIS3L-MAP2K1. The significant association for JMA testing at these loci in Europeans was primarily driven by SNP effects in both the lower and higher education strata $\left(4.40 \times 10^{-8} \leq P_{\operatorname{main}} \leq 1.35 \times 10^{-6}\right.$ and $7.61 \times 10^{-11} \leq P_{\text {main }} \leq 1.75 \times 10^{-6}, \quad$ respectively). $\quad \mathrm{SNP} \times$ education interaction was not significant $\left(P_{\text {int }} \geq 0.208\right)$. The estimated effect sizes of SNP effects on spherical equivalent were highly similar across education strata.

GEWIS in Asians. The JMA for spherical equivalent in 10,315 individuals from the Asians cohorts identified genome-wide significant association for three genes: AREG, GABRR1 and PDE10A $\left(P_{\mathrm{JMA}}<5.0 \times 10^{-8}\right.$; Table 3 and Fig. 2b). 
Table 1 | Characteristics of study participants.

\begin{tabular}{|c|c|c|c|c|c|c|}
\hline Study & $\mathbf{N}$ & Study year & Age (s.d.) & Age range & Male (\%) & Spherical equivalent \\
\hline \multicolumn{7}{|l|}{ Europeans $(n=40,036)$} \\
\hline ALIENOR & 509 & $2006-2008$ & $79.2(4.1)$ & $73-93$ & 43.2 & $0.98(1.98)$ \\
\hline ALSPAC & 1,865 & 1999-2000 & $45.9(4.5)$ & $32-59$ & 0 & $-0.76(2.16)$ \\
\hline AREDS & 1,842 & 1992 & $68.1(4.7)$ & $55-81$ & 41.0 & $0.54(2.15)$ \\
\hline BATS & 383 & 1992-2013 & $24.8(7.8)$ & $20-67$ & 41.3 & $-0.67(1.58)$ \\
\hline BMES & 1,896 & $1992-2009$ & $66.8(8.9)$ & $49-94$ & 43.8 & $0.58(1.94)$ \\
\hline CROATIA-Korcula & 807 & $2007-2008$ & $56.2(13.3)$ & $25-94$ & 34.9 & $-0.13(1.59)$ \\
\hline CROATIA-Split & 787 & $2008-2009$ & $51.9(13.0)$ & $25-80$ & 38.6 & $-1.27(1.59)$ \\
\hline DCCT & 1,057 & 1982-1993 & $35.4(5.8)$ & $25-49$ & 54.1 & $-1.47(1.80)$ \\
\hline EGCUT & 904 & $2002-2013$ & $56(17.0)$ & $25-99$ & 38.8 & $0.33(3.36)$ \\
\hline EPIC & 1,083 & 2004-2011 & $68.8(7.5)$ & $50-88$ & 43.8 & $0.34(2.27)$ \\
\hline ERF & 2,604 & $2002-2005$ & $48.9(14.4)$ & $25-87$ & 45.0 & $0.12(2.03)$ \\
\hline FES & 2,479 & 1973-1975/ 1989-1991 & $54.8(9.3)$ & $28-84$ & 55.3 & $0.27(2.37)$ \\
\hline FITSA & 188 & $2000-2001$ & $68.5(3.3)$ & $63-76$ & 0 & $1.44(2.08)$ \\
\hline GHS1 & 3,178 & $2007-2008$ & $55.3(10.9)$ & $35-74$ & 50.4 & $-0.38(2.47)$ \\
\hline GHS2 & 1,354 & 2008 & $54.6(10.8)$ & $36-74$ & 49.6 & $-0.39(2.51)$ \\
\hline KORA & 2326 & 2004-2006 & $55.1(11.8)$ & $35-84$ & 49.4 & $-0.26(2.18)$ \\
\hline OGP Talana & 456 & 2002 & $52.6(16.3)$ & $25-89$ & 57.3 & $-0.20(0.24)$ \\
\hline ORCADES & 1,124 & 2009 & $56.5(13.2)$ & $29-92$ & 39.1 & $0.10(2.07)$ \\
\hline RAINE & 348 & $2010-2012$ & $20.4(0.34)$ & $20-22$ & 49.1 & $0.03(1.29)$ \\
\hline RS1 & 5,702 & 1991-1993 & $68.7(8.7)$ & $55-99$ & 41.0 & $0.83(2.55)$ \\
\hline RS2 & 2,021 & $2000-2002$ & $64.3(7.9)$ & $55-95$ & 46.0 & $0.48(2.51)$ \\
\hline RS3 & 2,918 & 2006-2009 & $56.9(6.6)$ & $45-86$ & 44.0 & $-0.28(2.60)$ \\
\hline TwinsUK & 2,154 & $1998-2010$ & $53.8(11.4)$ & $25-84$ & 8.4 & $-0.96(2.78)$ \\
\hline WESDR & 561 & 1979-2007 & $31.7(7.0)$ & $25-65$ & 50.3 & $-1.65(2.07)$ \\
\hline YFS & 1,490 & 2011 & $41.9(5.0)$ & $34-49$ & 44.6 & $-1.09(2.16)$ \\
\hline \multicolumn{7}{|l|}{ Asians $(\mathrm{n}=10,315)$} \\
\hline BES & 589 & 2006-2011 & $62.1(8.5)$ & $50-90$ & 34.0 & $-0.06(1.86)$ \\
\hline Nagahama & 723 & $2008-2010$ & $49.2(15.2)$ & $30-74$ & 33.6 & $-1.93(2.46)$ \\
\hline SCES-610K & 1,710 & 2009-2011 & $57.5(7.0)$ & $44-84$ & 51.6 & $-0.72(2.69)$ \\
\hline SCES-OmniE & 543 & 2011-2012 & $59.3(8.9)$ & $46-83$ & 51.2 & $-0.89(2.74)$ \\
\hline SiMES & 2,256 & 2004-2006 & $46.8(10.2)$ & $40-80$ & 49.1 & $-0.03(1.81)$ \\
\hline SINDI & 2,088 & 2007-2009 & $55.8(8.8)$ & $43-84$ & 51.5 & $0.04(2.07)$ \\
\hline SP2-1M & 811 & 1992-1998 & $46.8(10.2)$ & $25-80$ & 62.3 & $-1.80(2.84)$ \\
\hline SP2-610 & 854 & 1992-1998 & $48.4(11.3)$ & $25-82$ & 19.6 & $-1.44(2.89)$ \\
\hline STARS & 741 & 2007-2009 & $38.5(5.2)$ & $26-58$ & 52.4 & $-2.80(2.85)$ \\
\hline
\end{tabular}

ALIENOR, antioxydants, lipids essentiels, nutrition et maladies oculaiRes; ALSPAC, avon longitudinal study of parents and children; AREDS, age-related eye disease study; BATS, Brisbane adolescent twins study; BMES, blue mountains eye study: DCCT, diabetes control and complications trial; EGCUT, estonian genome center of the university of Tartu; EPIC, EPIC-Norfolk eye study; ERF, erasmus rucphen family study; FES, Framingham eye study; FITSA, finnish twin study on aging; GHS, Gutenberg health study; KORA, cooperative health research in the region of Augsburg; OGP Talana, ogliastra genetic park, talana study; ORCADES, orkney complex disease study; RAINE, RAINE eye health study; RS, Rotterdam study; TwinsUK, Twins UK study; WESDR, Wisconsin epidemiologic study of diabetic retinopathy; YFS, young finns study; BES, Beijing eye study; SCES, Singapore Chinese eye study; SiMES, Singapore Malay eye study; SINDI, Singapore Indian eye study; SP2, Singapore prospective study program; STARS, strabismus, amblyopia and refractive error study in preschool singaporean children. s.d., standard deviation; age in years; spherical equivalent in dioptres.

Details of each study cohort are described in Supplementary Note 1.

$\mathrm{SNP} \times$ education interaction effects associated with spherical equivalent were observed at all three loci, with genetic effects significantly larger within participants who had a higher level of education compared with those with a lower education level: $A R E G\left(\mathrm{rs} 12511037, \beta_{\text {int }}=-0.89 \pm 0.14 \mathrm{D}, P_{\text {int }}=6.87 \times 10^{-11}\right)$, GABRR1 (rs13215566, $\left.\beta_{\text {int }}=-0.56 \pm 0.14 \mathrm{D}, P_{\text {int }}=8.48 \times 10^{-5}\right)$ and PDE10A (rs12206610, $\beta_{\text {int }}=-0.72 \pm 0.13 \mathrm{D}, \quad P_{\text {int }}=2.32$ $\times 10^{-8}$ ). The genotype and phenotype associations were highly significant in the higher education stratum (main genetic effects, $\left.1.97 \times 10^{-10} \leq P_{\text {main }} \leq 8.16 \times 10^{-8}\right)$ but were considerably weaker in the lower education stratum $\left(0.008 \leq P_{\text {main }} \leq 0.243\right)$. There was no evidence of inter-study heterogeneity at index SNPs within AREG, GABRR1 or PDE10A loci (Q-test: $P_{\text {het }} \geq 0.122$ ).

GABRR1 and PDE10A index SNPs were not associated with spherical equivalent in European samples, for either the JMA test, SNP main effect or SNP $\times$ education interaction (Table 3). AREG SNP rs12511037 was excluded in the meta-analysis of European studies after QC filtering; hence, a proxy SNP, rs1246413, in linkage disequilibrium (LD) with rs12511037 in Asians $\left(r^{2}=0.97\right)$ was tested but not associated with spherical equivalent
$\left(P_{\mathrm{JMA}}=0.527 ; \quad P_{\mathrm{int}}=0.176\right)$. The meta-regression including study-level characteristics as covariates in the model confirmed the heterogeneity between populations of European and Asian ancestry (GABRR1: $P=0.006 ; \quad P D E 10 A: \quad P=0.0419$; Supplementary Table 4). For PDE10A, besides ethnicity, average spherical equivalent of each study also explained the inter-study heterogeneity for the interaction effects $(P=0.025)$.

We examined whether the underlying assumption of $\mathrm{G} \times \mathrm{E}$ independence held at these three $\mathrm{G} \times \mathrm{E}$ interaction loci. We performed a meta-analysis of logistic regression analysis for education level on AREG SNP rs12511037, GABRR1 SNP rs13215566 and PDE10A SNP rs12296610, adjusting for age, gender and population stratification in Asian cohorts $(n=10,315)$. Our analysis did not reveal any significant associations between these loci and education level $(P \geq 0.102$, $P_{\text {het }} \geq 0.170$; Supplementary Table 5). Furthermore, the three loci were not associated with educational attainment in a large meta-analysis of GWAS recently conducted in European cohorts ${ }^{25}$. Thus, our $\mathrm{G} \times \mathrm{E}$ results are unlikely to be biased due to dependence between gene and education. 


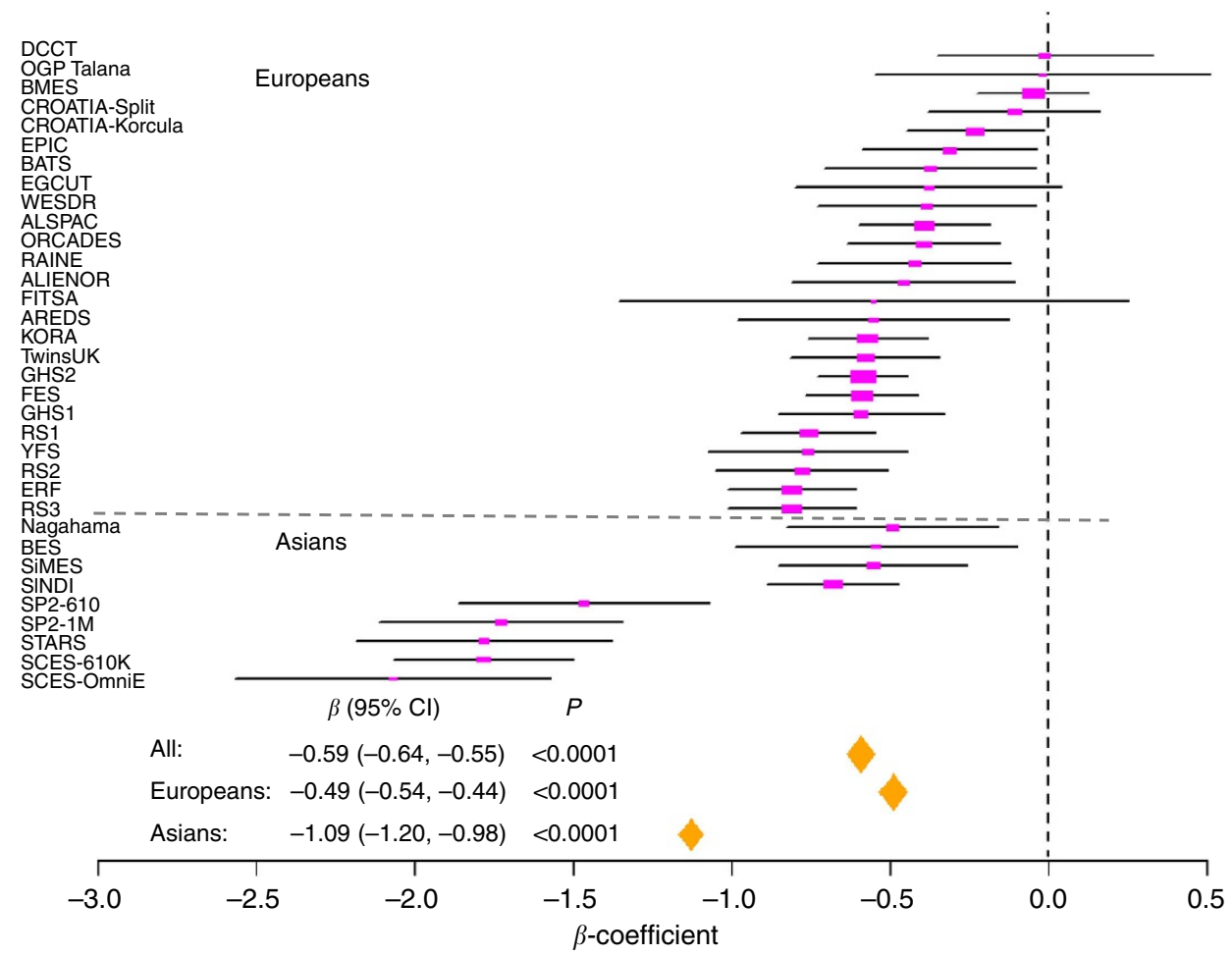

Figure 1 | Forest plot of education main effects on spherical equivalent across studies. The $\beta$-coefficient represents the differences of dioptres in refractive error comparing individuals in higher education group versus lower education group in Europeans $(n=40,036)$, Asians $(n=10,315)$ and all studies $(n=50,351)$. The studies are sorted by effect size of education on spherical equivalent within Europeans and Asians studies, respectively.

We also evaluated the association for spherical equivalent in Asian cohorts for four loci identified from European populations. Two of them showed significant associations in the JMA test: FAM150B-ACP1 $\left(P_{\mathrm{JMA}}=0.031\right)$ and DIS3L-MAP2K1 $\left(P_{\mathrm{JMA}}=0.0042\right.$; Table 2$)$. The SNP effect sizes in lower and higher education strata in Asians were similar at FAM150B$A C P 1$. The signal at the DIS3L-MAP2K1 locus was mainly driven by SNP $\times$ education interaction in Asians $\left(P_{\text {int }}=7.95 \times 10^{-4}\right)$, whereas the interaction effect was not statistically significant in Europeans $\left(P_{\text {int }}=0.208\right)$.

Combined GEWIS of all cohorts. We subsequently conducted a JMA in the combined data including both the European and Asian participants of all 34 studies. This analysis revealed two additional SNPs: ARID2-SNAT1 $\left(P_{\mathrm{JMA}}=4.38 \times 10^{-8}\right)$ and SLC14A2 $\left(P_{\mathrm{JMA}}=2.54 \times 10^{-8}\right)$. Both loci showed suggestive association with spherical equivalent in European cohorts, with the same direction of effect and similar effect sizes in Asian cohorts (Table 2). We also detected genome-wide significant associations with spherical equivalent for 17 known loci ${ }^{8}$ identified in our previous CREAM GWAS (Supplementary Table 3). The regional plots of the identified novel loci are presented in Supplementary Fig 2.

Gene and education interactions for GWAS known loci. We also evaluated the interactions between education and previously reported genetic association with spherical equivalent at 39 loci identified from recent two large GWAS studies ${ }^{7,8}$. Two $\mathrm{SNP} \times$ education interactions were nominally significant (Supplementary Table 6): TJP2 in Europeans (rs11145488, $P_{\text {int }}=6.91 \times 10^{-3}$ ) and SHISA6-DNAH9 in Asians (rs2969180, $\left.P_{\text {int }}=4.02 \times 10^{-3}\right)$. In general, the index SNPs tested at 39 loci had larger SNP $\times$ education interaction effect on spherical equivalent in Asians versus Europeans (meta-regression $P$ for fold changes $<0.001$; Supplementary Fig. 3). For 20 SNPs with the same direction of the interaction effect, the magnitudes of interaction effects were fourfold larger on average in Asians than in Europeans $(P=0.003)$.

Gene and near work interactions for three identified loci. High education levels may reflect an estimator for the greater accumulative effect of near work ${ }^{26,27}$. We thus examined whether there was evidence for SNP $\times$ near work interactions associated with spherical equivalent at the three loci (AREG, GABRR1 and $P D E 10 A)$ in paediatric cohorts $\left(\right.$ SCORM $^{28}$, Guangzhou Twins ${ }^{29}$ and ALSPAC ${ }^{30}$; combined $n=5,835$; Supplementary Table 7). Tentative support for a SNP $\times$ near work interaction was observed for PDE10A (rs12206610, $P_{\text {int }}=0.032, P_{\text {het }}=0.658$ ), with the stronger genetic effect in children spending more hours on reading, writing or compute use. Weaker support for an interaction was noted at GABRR1 (rs13215566, $P_{\text {int }}=0.309$, $\left.P_{\text {het }}=0.655\right)$, although the direction of meta-analysed interaction effect was largely consistent across paediatric studies with that observed in adults. We did not observe the interaction at AREG (rs12511037, $P_{\text {int }}=0.795, P_{\text {het }}=0.062$ ).

Gene expression in human tissues. Using the Ocular Tissue Database $^{31}$, we examined the expression of the associated genes in 20 normal human donor eyes. The majority of genes identified were expressed in human retina, sclera, choroid or retinal pigment epithelium (RPE) (Supplementary Table 8). Among these genes, GABRR1, ACP1 and SNAT1 had the highest expression in the retina. The Probe Logarithmic Intensity Error-normalized messenger RNA expression levels in the retina ranged from 121.66 to 236.69 . Of note, MAP2K1 was widely expressed in the retina, sclera and choroid/RPE. 
a

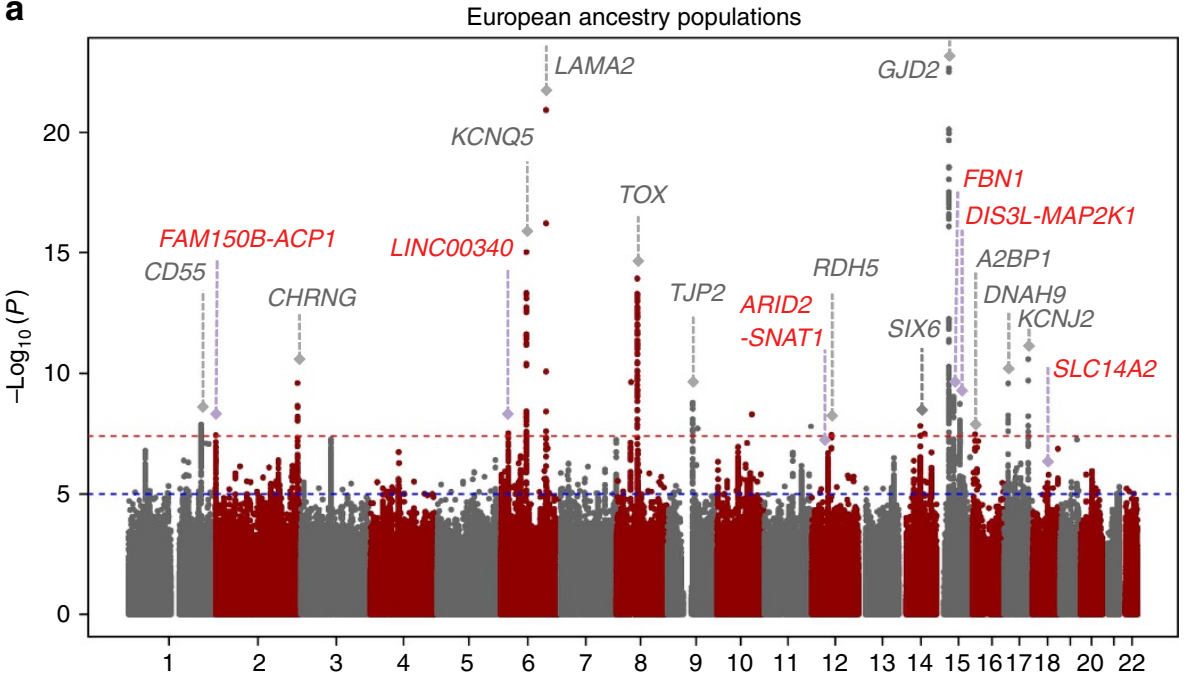

b

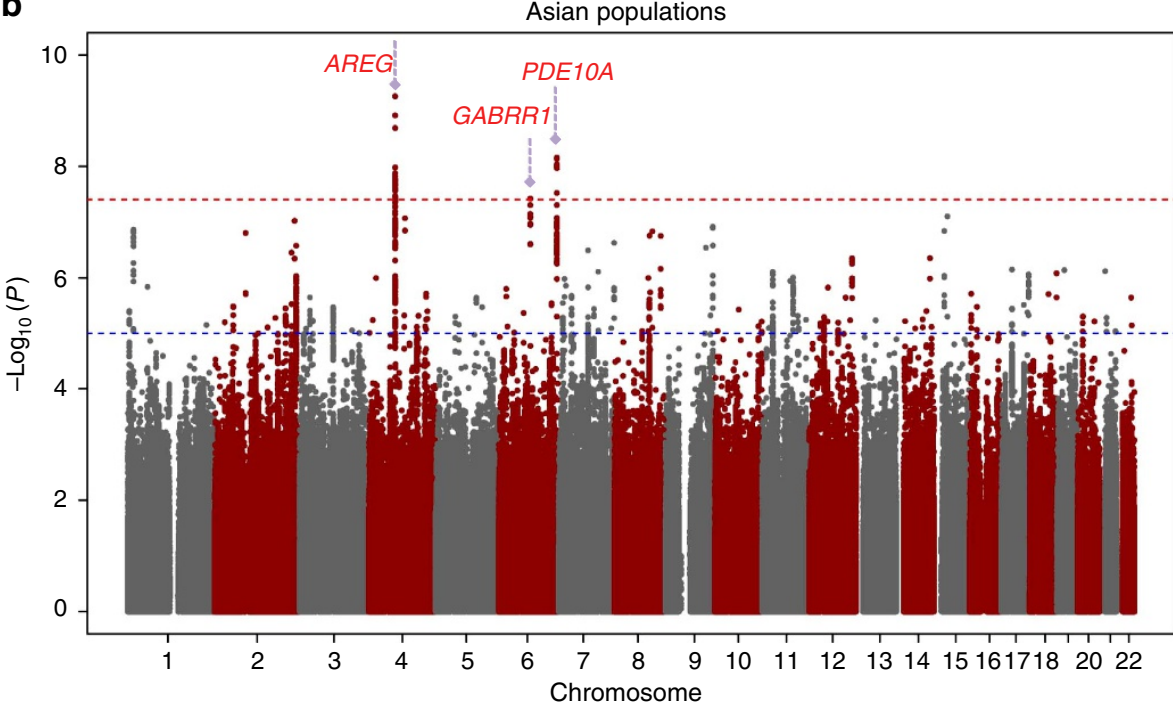

Figure 2 | Manhattan plots of $-\log _{10}(P)$ for the JMA on SNP main effects and SNP $\times$ education effects on spherical equivalent in (a) European ancestry populations and (b) Asian populations. The horizontal red line indicates the genome-wide significance level of $P_{\mathrm{JMA}}<5 \times 10^{-8}$. The horizontal blue line indicates the suggestive significance level of $P_{\mathrm{JMA}}<1 \times 10^{-5}$. Novel loci reaching genome-wide significance are labelled in red and known loci are in grey.

\section{Discussion}

This study represents the most comprehensive genome-wide scan of gene and education interactions to date, for refractive error. Here we identified novel genetic loci associated with refractive error by testing the joint contribution of SNP main effects and $\mathrm{SNP} \times$ education effects in large multi-ethnic populations. Three loci (AREG, GABRR1 and PDE10A) showed strong interactions with education in populations of Asian descent, with larger genetic effects within participants who had a higher level of education compared with those with a lower education level; no interactions achieved statistical significance in Europeans for top JMA associations or known myopic loci. Apart from confirming known associations at 17 previous published loci, we identified six new loci (FAM150B-ACP1, LINC00340, FBN1, DIS3L-MAP2K1, ARID2-SNAT1 and SLC14A2) significantly associated with spherical equivalent using the combined multi-racial cohort.

A recent meta-analysis of GWAS in multi-ethnic populations comprises 32 studies $(n=45,756)$ from CREAM and a large GWAS in Europeans $(n=45,771)$ have reported a total of 39 genetic loci associated with refractive phenotypes ${ }^{7,8}$. The current genome-wide meta-analysis included $\sim 5,000$ more subjects than the previous GWAS of main effects. We identified nine additional novel loci using the JMA approach. These loci can be placed within the biological context of the visually evoked signalling cascade that begins in the retina and mediates sclera remodelling ${ }^{32}$. The newly identified genes are involved in retinal neurotransmission (GABRR1 and SNAT1), extracellular matrix remodelling ( $F B N 1, M A P 2 K 1$ and $A R E G)$, circadian rhythm $(P D E 10 A)$ and platelet-derived growth factor receptor signalling (ACP1) (Supplementary Table 9). Network analysis revealed that most of the novel genes may tend to be co-expressed and co-localized with the known myopia susceptibility genes through multiple biological networks such as LAMA2, GJD2, RASGRF1, BMP3, RDH5, ZMAT4, RBFOX1, RDH5 and so on (Supplementary Fig. 4). Our data, in line with previous findings, substantiate the assumption of heterogeneity in the molecular mechanisms involved in refractive error and myopia.

Among the novel loci, GABRR1 on chromosome $6 \mathrm{q} 15(53 \mathrm{~kb})$, encoding $\mathrm{GABA}_{\mathrm{C}}$ receptor subunit $\rho 1$, is an interesting functional candidate suggestive of a role in myopia development. Modulation of synaptic plasticity via GABA-mediated inhibition would be well placed to alter the 'gain' of the visually guided 
Table 2 | Six genetic loci associated with spherical equivalent from the JMA in the European populations and combined analyses.

\begin{tabular}{|c|c|c|c|c|c|c|c|c|c|c|c|c|c|}
\hline \multirow[t]{2}{*}{ SNP (Chr:BP) } & \multirow[t]{2}{*}{ Gene } & \multirow[t]{2}{*}{ Allele } & \multirow[t]{2}{*}{ FREQ } & \multirow[t]{2}{*}{ Subgroup } & \multicolumn{3}{|c|}{ Europeans $(n=40,036)$} & \multicolumn{3}{|c|}{ Asians $(n=10,315)$} & \multicolumn{3}{|c|}{ All $(n=50,351)$} \\
\hline & & & & & $\beta$ & P-value & $P_{\text {het }}$ & $\beta$ & P-value & $P_{\text {het }}$ & $\beta$ & P-value & $\overline{P_{\text {het }}}$ \\
\hline \multirow{3}{*}{$\begin{array}{l}\text { rs60843830 } \\
(2: 286756)\end{array}$} & FAM150B- & $C / G$ & $0.66 / 0.74$ & JMA & & $3.71 \times 10^{-8}$ & 0.086 & & 0.031 & 0.980 & & $1.27 \times 10^{-9}$ & 0.395 \\
\hline & & & & $\begin{array}{l}\text { Lower } \\
\text { education }\end{array}$ & -0.11 & $4.73 \times 10^{-8}$ & & -0.09 & 0.010 & & -0.10 & $1.65 \times 10^{-9}$ & \\
\hline & & & & $\begin{array}{l}\text { Higher } \\
\text { education }\end{array}$ & -0.09 & $1.75 \times 10^{-6}$ & & -0.06 & 0.509 & & -0.09 & $9.83 \times 10^{-7}$ & \\
\hline \multirow[t]{3}{*}{$\begin{array}{l}\text { rs10946507 } \\
(6: 22100367)\end{array}$} & $\begin{array}{l}\text { LINCO0340 } \\
(6 p 223)\end{array}$ & $A / G$ & $0.47 / 0.16$ & JMA & & $3.07 \times 10^{-8}$ & 0.213 & & 0.433 & 0.396 & & $2.24 \times 10^{-8}$ & 0.249 \\
\hline & & & & $\begin{array}{l}\text { Lower } \\
\text { education }\end{array}$ & -0.08 & $7.08 \times 10^{-7}$ & & -0.04 & 0.313 & & -0.08 & $6.13 \times 10^{-7}$ & \\
\hline & & & & $\begin{array}{l}\text { Higher } \\
\text { education }\end{array}$ & -0.09 & $1.19 \times 10^{-8}$ & & -0.08 & 0.450 & & -0.09 & $1.20 \times 10^{-8}$ & \\
\hline \multirow{3}{*}{$\begin{array}{l}\text { rs8023401 } \\
(15: 48703823)\end{array}$} & FBN1 & $G / A$ & $0.87 / 0.95$ & JMA & & $1.66 \times 10^{-9}$ & 0.180 & & 0.572 & 0.979 & & $2.85 \times 10^{-9}$ & 0.495 \\
\hline & & & & $\begin{array}{l}\text { Lower } \\
\text { education }\end{array}$ & -0.15 & $4.40 \times 10^{-8}$ & & -0.06 & 0.304 & & -0.13 & $8.17 \times 10^{-8}$ & \\
\hline & & & & Higher & -0.16 & $7.61 \times 10^{-11}$ & & -0.03 & 0.828 & & -0.14 & $2.02 \times 10^{-9}$ & \\
\hline \multirow[t]{3}{*}{$\begin{array}{l}\text { rs16949788 } \\
(15: 66590037)\end{array}$} & $\begin{array}{l}\text { DIS3L- } \\
\text { MAP2K1 }\end{array}$ & $\mathrm{T} / \mathrm{C}$ & $0.91 / 0.94$ & $\begin{array}{l}\text { eaucation } \\
\text { JMA }\end{array}$ & & $1.34 \times 10^{-8}$ & 0.721 & & 0.0042 & 0.219 & & $2.19 \times 10^{-8}$ & 0.245 \\
\hline & & & & $\begin{array}{l}\text { Lower } \\
\text { education }\end{array}$ & -0.15 & $1.35 \times 10^{-6}$ & & 0.21 & 0.103 & & -0.13 & $4.88 \times 10^{-6}$ & \\
\hline & & & & $\begin{array}{l}\text { Higher } \\
\text { education }\end{array}$ & -0.17 & $1.89 \times 10^{-9}$ & & -0.59 & 0.014 & & -0.16 & $3.90 \times 10^{-9}$ & \\
\hline \multirow{3}{*}{$\begin{array}{l}\text { rs10880855 } \\
(12: 46144855)\end{array}$} & ARID2-SNAT1 & $\mathrm{T} / \mathrm{C}$ & $0.51 / 0.43$ & JMA & & $7.83 \times 10^{-7}$ & 0.790 & & 0.019 & 0.779 & & $4.38 \times 10^{-8}$ & 0.867 \\
\hline & & & & $\begin{array}{l}\text { Lower } \\
\text { education }\end{array}$ & -0.09 & $1.26 \times 10^{-7}$ & & -0.06 & 0.067 & & -0.09 & $8.42 \times 10^{-9}$ & \\
\hline & & & & $\begin{array}{l}\text { Higher } \\
\text { education }\end{array}$ & -0.07 & $1.60 \times 10^{-5}$ & & -0.16 & 0.033 & & -0.07 & $3.55 \times 10^{-6}$ & \\
\hline \multirow{3}{*}{$\begin{array}{l}\text { rs10853531 } \\
(18: 42824449)\end{array}$} & SLC14A2 & $G / A$ & $0.80 / 0.83$ & JMA & & $7.82 \times 10^{-6}$ & 0.052 & & 0.0023 & 0.812 & & $2.54 \times 10^{-8}$ & 0.111 \\
\hline & & & & $\begin{array}{l}\text { Lower } \\
\text { education }\end{array}$ & -0.11 & $1.27 \times 10^{-6}$ & & -0.15 & $9.01 \times 10^{-4}$ & & -0.11 & $3.38 \times 10^{-9}$ & \\
\hline & & & & $\begin{array}{l}\text { Higher } \\
\text { education }\end{array}$ & -0.08 & $2.12 \times 10^{-6}$ & & -0.11 & 0.288 & & -0.09 & $7.14 \times 10^{-6}$ & \\
\hline
\end{tabular}

$\beta, \beta$-coefficient corresponds to the effect in spherical equivalent (dioptres) for 1 additional copy of the risk allele in the higher or lower education group. FREQ, allele frequency of the risk allele in European/Asian cohorts; JMA, joint meta-analysis on SNP effect and SNP $\times$ education interaction effect on spherical equivalent; $P_{\text {het, }} P$-value for the test of heterogeneity at each SNP; SNP, single-nucleotide polymorphism. Allele, risk allele/other allele.

feedback system controlling refractive development ${ }^{33}$. The lead SNP rs13215566 in GABRR1, together with seven SNPs within the LD block $\left(r^{2} \geq 0.8\right)$, are intronic, potentially affecting regulatory motifs (such as $\mathrm{zfp} 128$ and $\mathrm{gcm} 1$ ) that may influence transcriptional regulation (Supplementary Table 10). The variant rs13215029, in LD $\left(r^{2}=1\right)$ with rs13215566, is associated with cis-acting expression of GABRR1 $\left(P=2.3 \times 10^{-4}\right)$ in skin tissues (Supplementary Table 9$)^{34}$. A recent pharmacological study provided evidence that retinal dopaminergic and GABAergic neurotransmitters play a substantial role in the modulation of refractive development in form-deprivation myopia ${ }^{35-37}$. Stone et $a .^{35}$ have reported that antagonists to GABA-A, -B and -C receptors inhibited form-deprivation myopia in chicks, with greatest effect in the equatorial dimension. GABA receptors, expressed in bipolar and ganglion neuron cells, also interact with dopamine pathways in the retina ${ }^{36}$. A recent proteomics study determined that levels of GABA transporter-1 were significantly reduced in myopic murine retina after atropine treatment, implying that GABA signalling is involved in the anti-myopic effects of atropine ${ }^{37}$. Altogether, these data suggest that $\mathrm{GABA}_{\mathrm{C}}$ receptor $\rho 1$ may regulate the development of myopia through functional feedback from RPE to neuron cells in the retina. Further studies are needed to investigate the effect of genetic deletion of GABRR1 on refractive eye development and the role of the GABAergic pathway in myopia development using gene knockout mice. Therefore, our result in humans is in line with animal experiments, supporting the notion that the GABAergic neurotransmitter signalling pathway in the retina could be a potential factor in the progression of myopia.
SNP rs10889855 on chromosome 6 is an intronic variant within the ARID2 gene (AT-rich interactive domain 2) and $500 \mathrm{~kb}$ downstream of SNAT1 (solute carrier family 38, member, alias SLC38A1). SNAT1 is a transporter of glutamine, a precursor of $\mathrm{GABA}^{38}$. It is also highly expressed in human retina. In our previous meta-analysis in $\mathrm{CREAM}^{8}$, we identified variants in another glutamate receptor gene GRIA4 (encoding glutamate receptor, ionotropic); altogether, current evidence supports the notion that retinal neurotransmitters GABA and glutamine may be involved in the refractive development.

The strongest association signal for gene and environment interactions was from rs 12511037 , located $14 \mathrm{~kb}$ downstream the AREG gene (amphiregulin). AREG is a ligand of the epidermal growth factor receptor promoting the growth of normal epithelial cells, which is critical for cell differentiation and proliferation such as regrowth of the wounded cornea ${ }^{39}$. A link has been found between the muscarinic acetylcholine receptors, dominant in myopia progression, and the epidermal growth factor receptor in muscarinic system ${ }^{40,41}$.

Another novel association, rs16949788 on chromosome 15, derives from a region that spans DIS3L and MAP2K1. MAP2K1 encodes mitogen-activated protein kinase 1 , which binds to muscarinic receptors during proliferation ${ }^{42}$ and inhibits the proliferation of human scleral fibroblasts exposed to all-trans retinoic acid ${ }^{43}$. All-trans retinoic acid is a modulator of ocular growth, inhibiting the proliferation of human scleral fibroblasts ${ }^{44}$.

FBN1 (Fibrillin 1), a member of the fibrillin family, encodes a large extracellular matrix glycoprotein. Mutations in FBN1 cause 


\begin{tabular}{|c|c|c|c|c|c|c|c|c|c|c|}
\hline \multirow[t]{2}{*}{ SNP (Chr:BP) } & \multirow[t]{2}{*}{ Gene } & \multirow[t]{2}{*}{ Allele } & \multirow[t]{2}{*}{ FREQ } & \multirow[t]{2}{*}{ Subgroup } & \multicolumn{3}{|c|}{$\begin{array}{c}\text { Asians } \\
(n=10,315)\end{array}$} & \multicolumn{3}{|c|}{$\begin{array}{c}\text { Europeans } \\
(n=40,036)\end{array}$} \\
\hline & & & & & $\beta$ & P-value & $P_{\text {het }}$ & $\beta$ & P-value & $P_{\text {het }}$ \\
\hline \multirow{3}{*}{$\begin{array}{l}r s 12511037^{\star} \\
(4: 75334864)\end{array}$} & AREG & $\mathrm{C} / \mathrm{T}$ & $0.91 / 0.95$ & Lower education & 0.07 & 0.243 & & -0.05 & 0.323 & \\
\hline & & & & $\mathrm{SNP} \times$ education & -0.89 & $6.87 \times 10^{-11}$ & 0.704 & 0.02 & 0.176 & 0.284 \\
\hline & & & & JMA & & $5.55 \times 10^{-10}$ & 0.405 & & 0.527 & 0.186 \\
\hline \multirow{2}{*}{$\begin{array}{l}\text { rs13215566 } \\
(6: 89918638)\end{array}$} & GABRR1 & $C / G$ & $0.94 / 0.84$ & Lower education & -0.13 & 0.030 & & -0.03 & 0.258 & \\
\hline & & & & $\begin{array}{l}\text { Higher } \\
\text { education }\end{array}$ & -0.68 & $1.46 \times 10^{-8}$ & & -0.01 & 0.817 & \\
\hline \multirow{2}{*}{$\begin{array}{l}\text { rs12206610 } \\
6: 166016800\end{array}$} & & & & SNP $\times$ education & -0.72 & $2.32 \times 10^{-8}$ & 0.920 & -0.002 & 0.421 & 0.111 \\
\hline & & & & JMA & & $9.21 \times 10^{-9}$ & 0.902 & & 0.954 & 0.305 \\
\hline
\end{tabular}

$\beta$ (higher education/lower education), $\beta$-coefficient corresponds to the effect in spherical equivalent (dioptres) for 1 additional copy of the effect allele in the higher/lower education group; $\beta$ (SNP $\times$ education), $\beta$-coefficient corresponds to the difference in spherical equivalent (dioptres) for 1 additional copy of the effect allele in the higher versus lower education group; FREQ, allele frequency of the effect allele in Asian/European cohorts; JMA, joint meta-analysis on SNP effect and SNP $\times$ education interaction effect on spherical equivalent; LD, linkage disequilibrium; $P_{\text {het, }} P$-value for the test of the effect alle

$\beta$ of heterogeneity; SNP, single-nucleotide polymorphism.
$\beta$ and $P$-values for SNP $\times$ education interaction were calculated by the meta-analysis of conducting a 1df Wald's test of single interaction parameter. Allele is listed as effect allele/other allele.

${ }^{*} \mathrm{SNP}$ rs12511037 was not present in European studies after quality control. Here we present the results of a proxy SNP rs $1246413(T / G$, frequency of risk allele $T=0.95)$ in LD with rs12511037 ( $\left.r^{2}=0.97\right)$.

Marfan's syndrome, a disorder of connective tissue affecting the ocular, skeletal and cardiovascular systems ${ }^{45}$. As a candidate gene for myopia, attempts to study its association with myopia previously produced inconclusive results ${ }^{46,47}$, probably owing, in part, to underpowered studies with insufficient sample sizes. Using data from a large multi-ethnic population, our results support the role of FBN1 in myopia development.

The genome-wide significant SNPs from the JMA approach did not exhibit any interactions with education in Europeans, in contrast to the significant interactive effect among Asians. In particular, the $\mathrm{G} \times \mathrm{E}$ interactions at $A R E G, G A B R R 1$ and $P D E 10 A$ were only evident in Asian populations. There are a number of possible reasons for the observed differences. First, the variation of LD patterns and joint effects of genetic variants might affect the transferability of $\mathrm{G} \times \mathrm{E}$ signals across populations. Similar LD patterns were seen at GABRR1 and PDE10A regions across populations whereas a long stretch of LD flanking AREG was present only in Asian populations (Supplementary Fig. 5). As the true causal variants transferrable across populations are probably not implicated in our study, the identified novel myopia risk loci provide a much-needed starting point for follow-up and functional downstream analyses. Second, we used education level in adults as a surrogate measure of the underlying risk factors for influencing refractive development. Ideally, near work intensity would be measured prospectively in children before the onset of myopia. Studies included in our analyses do not have additional data on relevant childhood exposures. Thus, the best available surrogate measure of cumulative near work exposure in adult cohorts is educational level. We nevertheless believe that education is a highly reliable proxy for the relevant exposures underlying refractive development and is universally associated with refractive error in our study. Third, the differences of $\mathrm{G} \times \mathrm{E}$ interactions in Asian versus Europeans may reflect quantitative differences in near work intensity during childhood. For example, 6- and 7- year-old children in England and Australia reported less near work activity outside of school $(1.0-2.3 \mathrm{~h} \text { per day })^{48,49}$ compared with children in Singapore and China $2.7-3.5 \mathrm{~h}$ per day $)^{50,51}$. A similar trend was observed in older children ${ }^{48,52-55}$. We thus speculate that the total exposure to near work activity may be greater in East Asians compared with European-derived populations with the same levels of education; hence, $\mathrm{G} \times \mathrm{E}$ interaction estimates would tend to be inflated in Asian populations compared with European groups. Fourth, other environmental factors such as outdoor activities could also interact with genes. East Asian children tend to have less exposure to outdoor activities compared with their European peers ${ }^{56}$. However, the majority of adult cohorts did not report time outdoors and thus could not be accounted for in the current study. Finally, the population mean of refractive error is less myopic in Europeans $(0.10 \mathrm{D})$ versus Asians $(-0.60 \mathrm{D})$. Of note, for the previously known myopia loci, the magnitudes of interaction effects were fourfold larger on average in Asians than in Europeans (Supplementary Fig. 2). The impact of $\mathrm{G} \times \mathrm{E}$ interactions may be seen at certain severity levels of myopia.

The risk alleles of rs12511037 in AREG, rs1321556 in GABRR1 and rs12206610 in PDE10A had no or weak influence on myopic shift in the lower education group compared with the higher education group. This suggests that the hereditary predisposition to myopia could be latent for the risk allele carriers, if they are less exposed to the myopiagenic environment associated with highlevel education. A lack of strong SNP $\times$ near work associations in children might be due to the inadequate statistical power in paediatric cohorts of relatively small sample sizes, or the possibility that environmental risk exposures other than near work might underlie the SNP $\times$ education interaction seen in the adult Asian samples.

In summary, we identified nine novel loci associated with refractive error in a large multi-ethnic cohort study by GEWIS approach. Our data provide evidence that specific genetic variants interact with education, to influence refractive development, and further support a role for GABA neurotransmitter signalling in myopia development. These findings provide promising 
candidate genes for follow-up work and may lead to new genetic targets for therapeutic interventions on myopia.

\section{Methods}

Study participants. Thirty-four studies from members of CREAM, comprising 40,036 individuals of European ancestry from 25 studies and 10,315 individuals of Asian ancestry from 9 studies, were made available for this analysis (Table 1 and Supplementary Note 1 ). Individuals aged $<20$ years were excluded and so were those who had undergone cataract surgery, laser or other intra-ocular procedures that could alter refraction. Many of these studies were also included in the previous CREAM GWAS on spherical equivalent ${ }^{8}$. All studies adhered to the tenets of the Declaration of Helsinki and were approved by their local research ethics committees. The exact names of the Institutional Research Board committees can be found under Supplementary Note 2. All participants provided a signed consent form before the start of the study.

Phenotyping and education levels. All participants underwent ophthalmological examinations (Supplementary Table 1). Non-cycloplegic refraction was measured by autorefraction and/or subjective refraction. Spherical equivalent was calculated as the sphere power plus half of the cylinder power for each eye. The mean spherical equivalent of the right and left eyes was used as a quantitative outcome. When data from only one eye was available, the spherical equivalent of that eye was used. For each study, the participants reported the highest level of education achieved or the years of schooling through a self-reported questionnaire, or in an interview.

We dichotomized education for all participants in each study. The higher education group consisted of those who had achieved the highest educational level of A-levels, high school (higher secondary education), vocational training (for example, diploma), university degree or those with $\geq 12$ years spent in formal education (beginning from first grade). Those who had achieved the highest educational level of O-level, middle school (lower secondary education) or those with $<12$ years of formal education were classified into the lower education group. If both number of formal study years and education levels were available in the cohort, we classified participants based on years of formal education. For the four cohorts of relatively young European participants (BATS, DCCT, RAINE and WESDR; total $n=2,349$ ), almost all of them had completed 12 or more years of schooling. We thus chose to categorize individuals with tertiary or university education as the higher education group in these studies. Sensitivity analysis excluding these four cohorts did not appreciably change our meta-analysis results.

Genotyping and imputation. Detailed information on the genotyping platforms and QC procedures for each study is provided in Supplementary Table 2 and Supplementary Note 1. Each study applied stringent QC filters for GWAS. In general, duplicate DNA samples, individuals with low call rate $(<95 \%)$, gender mismatch or ethnic outliers were excluded. SNPs were excluded if low genotyping call rate ( $>5 \%$ missingness), monomorphic SNPs, with minor allele frequency (MAF) $<1 \%$ or in Hardy-Weinberg disequilibrium $\left(P<10^{-6}\right)$. After QC filtering, the array genotypes of each study were imputed using the 1000 Genomes Project data as reference panels (build 37, phase 1 release, March 2012) with the software Minimac $^{57}$ or IMPUTE2 (ref. 58). Approximately six million SNPs that passed imputation quality thresholds (MACH: $r^{2}>0.5$ or IMPUTE info score $>0.5$ ) and with $\mathrm{MAF} \geq 5 \%$ were eligible for the meta-analysis (Supplementary Table 2).

Statistical models. For each study, a linear regression model for each genotyped or imputed SNP was constructed with the mean spherical equivalent as the outcome. We assumed an additive genetic model where the number of risk alleles is an ordinal variable $(0,1$ and 2$)$ for directly genotyped SNPs or a continuous variable of allele dosage probability ranging from 0 to 2 for imputed SNPs. The primary analytic model included SNP, education and SNP $\times$ education interaction term, as well as age and sex as covariates. Additional adjustments for the top principal components of genomic marker variations were performed in individual studies when applicable (that is, when there was evidence of population stratification)

We used the following additive genetic model to test for a joint effect of SNP $\left(\beta_{\mathrm{SNP}}\right)$ and $\mathrm{SNP} \times$ education interaction $\left(\beta_{\mathrm{SNP} \times \text { education }}\right)$ on mean spherical equivalent:

$Y=\beta_{0}+\beta_{\mathrm{SNP}} \times S N P+\beta_{\text {education }} \times$ education $+\beta_{\mathrm{SNP} \times \text { education }} \times S N P \times$ education $+\beta_{C} \times \operatorname{cov}+\varepsilon$

where $Y$ is the mean spherical equivalent and education is a dichotomous variable $(0=$ lower education group and $1=$ higher education group); cov is a set of covariates such as age, sex and first top five principal components when applicable. For family-based studies, the kinship matrix was estimated empirically from the SNP data and included as a random effect in the generalized mixed model ${ }^{59}$. To test an effect of SNP $\times$ education interaction, we assessed $\beta_{\mathrm{SNP} \times \text { education }}$ from equation (1).

The linear regression analyses in each study were conducted with Quickes or ProbABEL for the unrelated samples and MixABEL for family-based data. The command 'robust' was used in the above software to calculate the robust ('sandwich', Huber-White) s.e. of $\beta_{\mathrm{SNP}}$ and $\beta_{\mathrm{SNP}} \times$ education, and error covariance of $\beta$, to correct the potential inflation of false positive rate for the interaction $P$-value ${ }^{60}$

In addition, each study also tested the main effect of education on spherical equivalent by adjusting for age and gender using the linear regression model:

$$
Y=\beta_{0}+\beta_{\text {education }} \times \text { education }+\beta_{\mathrm{C}} \times \operatorname{cov}+\varepsilon
$$

where the definition of each variable is the same as in equation (1). We performed meta-analysis of the education effects on mean spherical equivalent in Europeans, Asians (Singapore Chinese versus others) and combined data using a fixed-effect model with inverse-variance weighting ( $\mathrm{R}$ package 'meta').

GEWIS join meta-analyses. We adopted the JMA approach ${ }^{17,61}$, to simultaneously test both SNP main effects and SNP $\times$ education interactions for spherical equivalent with a fixed-effect model, using SNP and SNP $\times$ education regression coefficients $\left(\beta_{\mathrm{SNP}}\right.$ and $\beta_{\mathrm{SNP}} \times$ education, respectively) and a $\beta$ 's covariance matrix from each study. A Wald's statistic, following a $\chi^{2}$-distribution with two degrees of freedom, was used to test the joint significance of the $\beta_{\mathrm{SNP}}$ and $\beta_{\text {SNP } \times \text { education. The JMA was performed with METAL }}{ }^{62}$, as previously described by Manning et al. ${ }^{61}$. A Cochran's $Q$-test was used to assess heterogeneity of the $\beta$-coefficients across studies for the SNP and interaction effects. To test for interaction between the SNP and education, we conducted a secondary metaanalysis of the SNP $\times$ education interaction effects for spherical equivalent $\left(\beta_{\mathrm{SNP} \times \text { education }}\right.$, one degree of freedom), with a fixed-effects model using inversevariance weighting in METAL; this is a traditional meta-analysis to investigate $\mathrm{SNP} \times$ education interactions per se. Effects and s.e. of the SNP effect on spherical equivalent in the lower education group $\left(\beta_{\mathrm{SNP}}\right)$ and higher education group $\left(\beta_{\mathrm{SNP}}+\beta_{\mathrm{SNP} \times \text { education }}\right)$ were derived from the JMA output ${ }^{61}$. We used the $P$-value of $5 \times 10^{-8}$ as a significant threshold for JMA test. For the SNP and SNP $\times$ education effects for the identified top loci, the $P$-value threshold for significance was set at $0.0055=0.05 / 9$ ( 9 index SNPs underlying analyses).

We performed a meta-regression to explore sources of heterogeneity in our meta-analysis for three loci showing $\mathrm{G} \times \mathrm{E}$ interactions ( $\mathrm{R}$ package 'metafor'). Meta-regression included the following study-specific variables as covariates: study sample size, proportion of individuals in the higher education group, average spherical equivalent, education main effects on spherical equivalent (higher education level versus lower), ethnicity (Asian versus European), study design (independent samples versus family-based studies), study year and average age Meta-regression was also conducted to test the fold changes of the interaction $\beta$-coefficients in Asians versus Europeans for the 39 known myopia loci.

The study-specific genomic control inflation factors $\lambda_{\mathrm{gc}}$ for the joint test for SNP and interaction terms ranged from 1.009 to 1.125 with an average of 1.019 (Supplementary Table 2), calculated by the ratio of the observed median $\chi^{2}$ divided by the expected median of the $2 \mathrm{df} \chi^{2}$-distribution (1.382). Genomic control correction was applied to each individual study ${ }^{63}$. For studies of small sample sizes $(n<500)$ with $\lambda_{\mathrm{gc}}>1.05$, we further, before starting the meta-analysis, excluded SNPs showing significant joint $P$-value $<1 \times 10^{-5}$ but neither the SNP nor $\mathrm{SNP} \times$ education effects supported such an association. Quantile-quantile plots showed only modest inflation of the test statistics in the JMA test (Europeans: $\lambda_{\mathrm{gc}}=1.081$; Asians: $\lambda_{\mathrm{gc}}=1.053$; Combined: $\lambda_{\mathrm{gc}}=1.092$; Supplementary Fig. 1 ), similar to previous GEWIS studies with comparable sample sizes ${ }^{18,19}$. We excluded a small number of markers in the meta-analysis with $P_{\text {het }}<0.0001$. The $\lambda_{\mathrm{gc}}$ for the $\mathrm{SNP} \times$ education interaction term in the individual studies ranged from 1.01 to 1.08 , indicating little evidence of test statistic inflation on SNP $\times$ education effect for each study.

\section{Annotation of genetic variants and gene expression in humans. The} coordinates and variant identifiers are reported on the NCBI B37 (hg19) genome build and annotated using UCSC Genome Browser ${ }^{64}$. We identified variants within each of the LD blocks $\left(r^{2} \geq 0.8\right)$ in European and Asian populations of the 1000 Genomes Project (100 kb flanking the top SNP at each locus), to apply functional annotations of transcription regulation using HaploReg ${ }^{65}$ and Encyclopedia of DNA Elements ${ }^{66}$ data. We also generated funcational association and coexpression network using GeneMANIA ${ }^{67}$, to determine whether the disease-related genes identified in this study and previous $\mathrm{GWAS}^{7,8}$ are functionally connected.

To assess gene expression in human tissues, we examined the Ocular Tissue Database and the EyeSAGE database ${ }^{31,68}$. The estimated gene and exome-level abundances are available online. Normalization of gene expression used the Probe Logarithmic Intensity Error method with genomic control-background correction $^{31}$. Relationships between genotype and cis regulation of gene expression levels (Supplementary Table 9) were assessed using expression quantitative trait locus associations in multiple human tissues from UK samples ${ }^{34}$, as well as gene expression profiles obtained from GTExPortal database ${ }^{69}$

\section{References}

1. Morgan, I. G., Ohno-Matsui, K. \& Saw, S. M. Myopia. Lancet 379, 1739-1748 (2012).

2. Saw, S. M., Gazzard, G., Shih-Yen, E. C. \& Chua, W. H. Myopia and associated pathological complications. Ophthalmic Physiol. Opt. 25, 381-391 (2005).

3. Wojciechowski, R. Nature and nurture: the complex genetics of myopia and refractive error. Clin. Genet. 79, 301-320 (2011). 
4. Sanfilippo, P. G., Hewitt, A. W., Hammond, C. J. \& Mackey, D. A. The heritability of ocular traits. Surv. Ophthalmol. 55, 561-583 (2010).

5. Wong, T. Y. et al. Prevalence and risk factors for refractive errors in adult Chinese in Singapore. Invest. Ophthalmol. Vis. Sci. 41, 2486-2494 (2000).

6. Kempen, J. H. et al. The prevalence of refractive errors among adults in the United States, Western Europe, and Australia. Arch. Ophthalmol. 122, 495-505 (2004).

7. Kiefer, A. K. et al. Genome-wide analysis points to roles for extracellular matrix remodeling, the visual cycle, and neuronal development in myopia. PLoS Genet. 9, e1003299 (2013).

8. Verhoeven, V. J. et al. Genome-wide meta-analyses of multiancestry cohorts identify multiple new susceptibility loci for refractive error and myopia. Nat. Genet. 45, 314-318 (2013).

9. Verhoeven, V. J. et al. Education influences the role of genetics in myopia. Eur. J. Epidemiol. 28, 973-980 (2013).

10. Fan, Q. et al. Education influences the association between genetic variants and refractive error: a meta-analysis of five Singapore studies. Hum. Mol. Genet. 23, 546-554 (2014)

11. Goss, D. A. Nearwork and myopia. Lancet 356, 1456-1457 (2000).

12. Gwiazda, J., Thorn, F. \& Held, R. Accommodation, accommodative convergence, and response $\mathrm{AC} / \mathrm{A}$ ratios before and at the onset of myopia in children. Optom. Vis. Sci. 82, 273-278 (2005).

13. Strauss, O. The retinal pigment epithelium in visual function. Physiol. Rev. 85, 845-881 (2005).

14. Rymer, J. \& Wildsoet, C. F. The role of the retinal pigment epithelium in eye growth regulation and myopia: a review. Vis. Neurosci. 22, 251-261 (2005).

15. Cottriall, C. L., Brew, J., Vessey, K. A. \& McBrien, N. A. Diisopropylfluorophosphate alters retinal neurotransmitter levels and reduces experimentally-induced myopia. Naunyn Schmiedebergs Arch. Pharmacol. 364, 372-382 (2001)

16. Thomas, D. Gene--environment-wide association studies: emerging approaches. Nat. Rev. Genet. 11, 259-272 (2010).

17. Aschard, H., Hancock, D. B., London, S. J. \& Kraft, P. Genome-wide metaanalysis of joint tests for genetic and gene-environment interaction effects. Hum. Hered. 70, 292-300 (2010).

18. Manning, A. K. et al. A genome-wide approach accounting for body mass index identifies genetic variants influencing fasting glycemic traits and insulin resistance. Nat. Genet. 44, 659-669 (2012).

19. Hancock, D. B. et al. Genome-wide joint meta-analysis of SNP and SNP-bysmoking interaction identifies novel loci for pulmonary function. PLoS Genet. 8, e1003098 (2012).

20. Parssinen, O. et al. Heritability of spherical equivalent: a population-based twin study among 63- to 76-year-old female twins. Ophthalmology 117, 1908-1911 (2010).

21. Biino, G. et al. Ocular refraction: heritability and genome-wide search for eye morphometry traits in an isolated Sardinian population. Hum. Genet. 116, 152-159 (2005)

22. Age-Related Eye Disease Study Research, G. A randomized, placebo-controlled, clinical trial of high-dose supplementation with vitamins $\mathrm{C}$ and $\mathrm{E}$, beta carotene, and zinc for age-related macular degeneration and vision loss: AREDS report no. 8. Arch. Ophthalmol. 119, 1417-1436 (2001).

23. Foong, A. W. et al. Rationale and methodology for a population-based study of eye diseases in Malay people: The Singapore Malay eye study (SiMES). Ophthalmic Epidemiol. 14, 25-35 (2007).

24. Nakata, I. et al. Prevalence and characteristics of age-related macular degeneration in the Japanese population: the Nagahama study. Am. J. Ophthalmol. 156, 1002-1009 e2 (2013).

25. Rietveld, C. A. et al. GWAS of 126,559 individuals identifies genetic variants associated with educational attainment. Science 340, 1467-1471 (2013).

26. Lee, Y. Y., Lo, C. T., Sheu, S. J. \& Lin, J. L. What factors are associated with myopia in young adults? A survey study in Taiwan Military Conscripts. Invest. Ophthalmol. Vis. Sci. 54, 1026-1033 (2013).

27. Wong, L., Coggon, D., Cruddas, M. \& Hwang, C. H. Education, reading, and familial tendency as risk factors for myopia in Hong Kong fishermen. J. Epidemiol. Community Health 47, 50-53 (1993).

28. Saw, S. M., Hong, C. Y., Chia, K. S., Stone, R. A. \& Tan, D. Nearwork and myopia in young children. Lancet 357, 390 (2001).

29. Zheng, Y., Ding, X., Chen, Y. \& He, M. The Guangzhou Twin Project: an update. Twin Res. Hum. Genet. 16, 73-78 (2013).

30. Fraser, A. et al. Cohort Profile: the Avon Longitudinal Study of Parents and Children: ALSPAC mothers cohort. Int. J. Epidemiol. 42, 97-110 (2013).

31. Wagner, A. H. et al. Exon-level expression profiling of ocular tissues. Exp. Eye Res. 111, 105-111 (2013).

32. Young, T. L., Metlapally, R. \& Shay, A. E. Complex trait genetics of refractive error. Arch. Ophthalmol. 125, 38-48 (2007).

33. Chen, Y. P. et al. Selective breeding for susceptibility to myopia reveals a gene-environment interaction. Invest. Ophthalmol. Vis. Sci. 52, 4003-4011 (2011)
34. Grundberg, E. et al. Mapping cis- and trans-regulatory effects across multiple tissues in twins. Nat. Genet. 44, 1084-1089 (2012).

35. Stone, R. A. et al. GABA, experimental myopia, and ocular growth in chick. Invest. Ophthalmol. Vis. Sci. 44, 3933-3946 (2003).

36. Schmid, K. L., Strasberg, G., Rayner, C. L. \& Hartfield, P. J. The effects and interactions of GABAergic and dopaminergic agents in the prevention of form deprivation myopia by brief periods of normal vision. Exp. Eye Res. 110, 88-95 (2013).

37. Barathi, V. A. et al. Involvement of GABA transporters in atropine-treated myopic retina as revealed by iTRAQ quantitative proteomics. J. Proteome Res. (2014).

38. Albrecht, J., Sidoryk-Wegrzynowicz, M., Zielinska, M. \& Aschner, M. Roles of glutamine in neurotransmission. Neuron Glia. Biol. 6, 263-276 (2010).

39. Wang, K., Yamamoto, H., Chin, J. R., Werb, Z. \& Vu, T. H. Epidermal growth factor receptor-deficient mice have delayed primary endochondral ossification because of defective osteoclast recruitment. J. Biol. Chem. 279, 53848-53856 (2004).

40. Tsai, W., Morielli, A. D. \& Peralta, E. G. The m1 muscarinic acetylcholine receptor transactivates the EGF receptor to modulate ion channel activity. EMBO J. 16, 4597-4605 (1997).

41. Duncan, G. \& Collison, D. J. Role of the non-neuronal cholinergic system in the eye: a review. Life Sci. 72, 2013-2019 (2003).

42. Wotta, D. R., Wattenberg, E. V., Langason, R. B. \& el-Fakahany, E. E. M1, M3 and M5 muscarinic receptors stimulate mitogen-activated protein kinase. Pharmacology 56, 175-186 (1998).

43. Huo, L. et al. All-trans retinoic acid modulates mitogen-activated protein kinase pathway activation in human scleral fibroblasts through retinoic acid receptor beta. Mol. Vis. 19, 1795-1803 (2013).

44. Arumugam, B. \& McBrien, N. A. Muscarinic antagonist control of myopia: evidence for M4 and M1 receptor-based pathways in the inhibition of experimentally-induced axial myopia in the tree shrew. Invest. Ophthalmol. Vis. Sci. 53, 5827-5837 (2012).

45. Dietz, H. C. \& Pyeritz, R. E. Mutations in the human gene for fibrillin-1 (FBN1) in the Marfan syndrome and related disorders. Hum. Mol. Genet. 1799-1809 (1995).

46. Farbrother, J. E. et al. Linkage analysis of the genetic loci for high myopia on $18 \mathrm{p}, 12 \mathrm{q}$, and $17 \mathrm{q}$ in 51 U.K. families. Invest. Ophthalmol. Vis. Sci. 45, 2879-2885 (2004).

47. Yip, S. P. et al. A DNA pooling-based case-control study of myopia candidate genes COL11A1, COL18A1, FBN1, and PLOD1 in a Chinese population. Mol. Vis. 17, 810-821 (2011).

48. French, A. N., Morgan, I. G., Mitchell, P. \& Rose, K. A. Risk factors for inciden myopia in Australian schoolchildren: the Sydney adolescent vascular and eye study. Ophthalmology 120, 2100-2108 (2013).

49. Guggenheim, J. A. et al. Time outdoors and physical activity as predictors of incident myopia in childhood: a prospective cohort study. Invest. Ophthalmol. Vis. Sci. 53, 2856-2865 (2012).

50. Saw, S. M. et al. Nearwork in early-onset myopia. Invest. Ophthalmol. Vis. Sci. 43, 332-339 (2002)

51. Guo, Y. et al. Myopic shift and outdoor activity among primary school children: one-year follow-up study in Beijing. PLOS ONE 8, e75260 (2013).

52. Mutti, D. O., Mitchell, G. L., Moeschberger, M. L., Jones, L. A. \& Zadnik, K. Parental myopia, near work, school achievement, and children's refractive error. Invest. Ophthalmol. Vis. Sci. 43, 3633-3640 (2002).

53. Haworth, C. M., Davis, O. S. \& Plomin, R. Twins Early Development Study (TEDS): a genetically sensitive investigation of cognitive and behavioral development from childhood to young adulthood. Twin Res. Hum. Genet. 16, 117-125 (2013).

54. Lin, Z. et al. Near work, outdoor activity, and their association with refractive error. Optom. Vis. Sci. 91, 376-382 (2014).

55. Lu, B. et al. Associations between near work, outdoor activity, and myopia among adolescent students in rural China: the Xichang Pediatric Refractive Error Study report no. 2. Arch. Ophthalmol. 127, 769-775 (2009).

56. French, A. N., Ashby, R. S., Morgan, I. G. \& Rose, K. A. Time outdoors and the prevention of myopia. Exp. Eye Res. 114, 58-68 (2013).

57. Howie, B., Fuchsberger, C., Stephens, M., Marchini, J. \& Abecasis, G. R. Fast and accurate genotype imputation in genome-wide association studies through pre-phasing. Nat. Genet. 44, 955-959 (2012).

58. Howie, B. N., Donnelly, P. \& Marchini, J. A flexible and accurate genotype imputation method for the next generation of genome-wide association studies PLoS Genet. 5, e1000529 (2009).

59. Aulchenko, Y. S., Ripke, S., Isaacs, A. \& van Duijn, C. M. GenABEL: an R library for genome-wide association analysis. Bioinformatics 23, 1294-1296 (2007).

60. Voorman, A., Lumley, T., McKnight, B. \& Rice, K. Behavior of QQ-plots and genomic control in studies of gene-environment interaction. PLOS ONE 6, e19416 (2011). 
61. Manning, A. K. et al. Meta-analysis of gene-environment interaction: joint estimation of SNP and SNP $\times$ environment regression coefficients. Genet. Epidemiol. 35, 11-18 (2011).

62. Willer, C. J., Li, Y. \& Abecasis, G. R. METAL: fast and efficient meta-analysis of genomewide association scans. Bioinformatics 26, 2190-2191 (2010).

63. Devlin, B. \& Roeder, K. Genomic control for association studies. Biometrics 55, 997-1004 (1999).

64. Kent, W. J. et al. The human genome browser at UCSC. Genome Res. 12, 996-1006 (2002).

65. Ward, L. D. \& Kellis, M. HaploReg: a resource for exploring chromatin states, conservation, and regulatory motif alterations within sets of genetically linked variants. Nucleic Acids Res. 40, D930-D934 (2012).

66. Consortium, E. P. et al. An integrated encyclopedia of DNA elements in the human genome. Nature 489, 57-74 (2012).

67. Warde-Farley, D. et al. The GeneMANIA prediction server: biological network integration for gene prioritization and predicting gene function. Nucleic Acids Res. 38, W214-W220 (2010).

68. Bowes Rickman, C. et al. Defining the human macula transcriptome and candidate retinal disease genes using EyeSAGE. Invest. Ophthalmol. Vis. Sci. 47, 2305-2316 (2006).

69. Rivas, M. A. et al. Human genomics. Effect of predicted protein-truncating genetic variants on the human transcriptome. Science 348, 666-669 (2015).

\section{Acknowledgements}

We gratefully thank all the participants or volunteers who participated in the studies. A full list of funding information and acknowledgements by study can be found in the Supplementary Note 2 .

\section{Authors contributions}

Q.F., V.J.M.V., R.W., C.-Y.C, C.J.H., C.C.W.K. and S.-M.S. conceived the project. Q.F., V.J.M.V., R.W., V.A.B., P.G.H., J.A.G., R.H., V.V., A.P.K., S.M.H., T.L., Y.L., T.H., J.X.,
C.D.,L.P., J.W.,P.G., C.V., M.M., A.W.H., X.G., J.M., R.P.I., C.L.S., C.B., A.D.P., J.E.B.-W., Y.-Y.T., X.W., X.W., E.T.-H.B., C.E.H.H. and W.L. performed analyses. J.E.H., K.M.W., K.Y., O.P., H.C., I.R., Z.V., J.F.W., P.K.J, G.M., B.S.P., D.M.E., T.-H.S.-A., A.M., A.C.-G., M.B., O.R., M.K., I.S., T.Z., T.M., J.S.R., C.G., M.P., E.M.v.L., N.A., A.G.U., F.R., A.H., J.R.V., Y.X.W., M.K.I., C.S., P.G., V.T., L.Z., R.W.B., R.S., E.-S.T., E.V., E.M., C.-C.K., C.H., R.N.L., P.J.F., B.E.K.K., R.K., H.-S.W., P.M., A.M., T.A., T.L.Y., M.H., O.P., C.M.v.D., J.J.W., C.W., J.B.J.,Y.-Y.T., D.A.M., K.O., N.Y., N.P., T.-Y.W, P.N.B., D.S., C.-Y.C. and C.J.H. were responsible for collecting clinic data and performing genotyping in each study. Q.F., V.J.M.V., R.W., C.C.W.K. and S.-M.S. drafted the paper. P.G.H., V.A.B., J.A.G., V.V., R.H., A.P.K., A.D.P., S.M.H., T.Y., J.J.W., T.-Y.W., P.N.B., C.J.H. and C.-Y.C. critically reviewed the manuscript.

\section{Additional information}

Supplementary Information accompanies this paper at http://www.nature.com/ naturecommunications

Competing financial interests: The authors declare no competing financial interests.

Reprints and permission information is available online at http://npg.nature.com/ reprintsandpermissions/

How to cite this article: Fan, Q. et al. Meta-analysis of gene-environment-wide association scans accounting for education level identifies additional loci for refractive error. Nat. Commun. 7:11008 doi: 10.1038/ncomms11008 (2016).

This work is licensed under a Creative Commons Attribution 4.0 International License. The images or other third party material in this article are included in the article's Creative Commons license, unless indicated otherwise in the credit line; if the material is not included under the Creative Commons license, users will need to obtain permission from the license holder to reproduce the material. To view a copy of this license, visit http://creativecommons.org/licenses/by/4.0/

Qiao Fan ${ }^{1,2, \star}$, Virginie J.M. Verhoeven ${ }^{3,4, \star}$, Robert Wojciechowski ${ }^{5,6, \star}$, Veluchamy A. Barathi ${ }^{1,2,7, \star}$, Pirro G. Hysi $^{8, *}$, Jeremy A. Guggenheim ${ }^{9}$, René Höhn ${ }^{10,11}$, Veronique Vitart ${ }^{12}$, Anthony P. Khawaja ${ }^{13}$, Kenji Yamashiro ${ }^{14}$, S. Mohsen Hosseini ${ }^{15}$, Terho Lehtimäki ${ }^{16}, Y_{i}$ Lu $^{17}$, Toomas Haller ${ }^{18}$, Jing Xie ${ }^{19}$, Cécile Delcourt ${ }^{20,21}$, Mario Pirastu22, Juho Wedenoja ${ }^{23,24}$, Puya Gharahkhani ${ }^{17}$, Cristina Venturini ${ }^{8,25}$, Masahiro Miyake ${ }^{14}$, Alex W. Hewitt ${ }^{19,26}$, Xiaobo Guo ${ }^{27}$, Johanna Mazur ${ }^{28}$, Jenifer E. Huffman ${ }^{12}$, Katie M. Williams ${ }^{8,29}$, Ozren Polasek ${ }^{30}$, Harry Campbell ${ }^{31}$, Igor Rudan ${ }^{31}$, Zoran Vatavuk ${ }^{32}$, James F. Wilson ${ }^{31}$, Peter K. Joshi ${ }^{31}$, George McMahon 33,34, Beate St Pourcain 33,34,35, David M. Evans 33,34,36, Claire L. Simpson 5,37, Tae-Hwi Schwantes-An ${ }^{5}$, Robert P. Igo ${ }^{38}$, Alireza Mirshahi10,39, Audrey Cougnard-Gregoire20,21, Céline Bellenguez ${ }^{40,41,42}$, Maria Blettner ${ }^{28}$, Olli Raitakari ${ }^{43,44}$, Mika Kähönen ${ }^{45}$, Ilkka Seppälä16, Tanja Zeller ${ }^{46}$, Thomas Meitinger 47,48 , Consortium for Refractive Error and Myopia (CREAM) ${ }^{\dagger}$, Janina S. Ried ${ }^{49}$, Christian Gieger ${ }^{49}$, Laura Portas ${ }^{22}$, Elisabeth M. van Leeuwen ${ }^{4}$, Najaf Amin ${ }^{4}$, André G. Uitterlinden ${ }^{4,50,51}$, Fernando Rivadeneira 4,50,51, Albert Hofman ${ }^{4,51}$, Johannes R. Vingerling ${ }^{3}$, Ya Xing Wang ${ }^{52}$, Xu Wang53, Eileen Tai-Hui Boh ${ }^{53}$, M. Kamran Ikram¹,2, Charumathi Sabanayagam ${ }^{1,2}$, Preeti Gupta ${ }^{1}$, Vincent Tan', Lei Zhou', Candice E.H. Ho ${ }^{1}$, Wan'e Lim ${ }^{7}$, Roger W. Beuerman ${ }^{1,2,7}$, Rosalynn Siantar, ${ }^{1,54}$, E-Shyong Tai ${ }^{2,53,55}$, Eranga Vithana',2,7, Evelin Mihailov' ${ }^{18}$, Chiea-Chuen Khor 1,53,56, Caroline Hayward ${ }^{12}$, Robert N. Luben ${ }^{13}$, Paul J. Foster ${ }^{57,58}$, Barbara E.K. Klein ${ }^{59}$, Ronald Klein ${ }^{59}$, Hoi-Suen Wong ${ }^{15}$, Paul Mitchell ${ }^{60}$, Andres Metspalu ${ }^{18}$, Tin Aung 1,7, Terri L. Young 61 , Mingguang He ${ }^{62}$, Olavi Pärssinen ${ }^{63,64}$, Cornelia M. van Duijn ${ }^{4}$, Jie Jin Wang60, Cathy Williams ${ }^{34}$, Jost B. Jonas ${ }^{52,65}$, Yik-Ying Te0 53,56,66, David A. Mackey 26,67, Konrad Oexle ${ }^{48}$, Nagahisa Yoshimura ${ }^{14}$, Andrew D. Paterson ${ }^{15}$, Norbert Pfeiffer ${ }^{10}$, Tien-Yin Wong $1,2,7,53$, Paul N. Baird ${ }^{19}$, Dwight Stambolian ${ }^{68}$, Joan E. Bailey-Wilson ${ }^{5}$, Ching-Yu Cheng ${ }^{1,2,7, \star \star}$, Christopher J. Hammond ${ }^{8,29, \star \star}$, Caroline C.W. Klaver ${ }^{3,4, \star \star} \&$ Seang-Mei Saw ${ }^{1,2,7,53, \star \star}$ 
${ }^{1}$ Singapore Eye Research Institute, Singapore National Eye Centre, Singapore 169856, Singapore. ${ }^{2}$ Duke-NUS Medical School, Singapore 169857 , Singapore. ${ }^{3}$ Department of Ophthalmology, Erasmus Medical Center, 3000 CA Rotterdam, The Netherlands. ${ }^{4}$ Department of Epidemiology, Erasmus Medical Center, 3000 CA Rotterdam, The Netherlands. ${ }^{5}$ Computational and Statistical Genomics Branch, National Human Genome Research Institute, National Institutes of Health, Baltimore, Maryland 21224, USA. ${ }^{6}$ Department of Epidemiology, Johns Hopkins Bloomberg School of Public Health, Baltimore, Maryland 20205, USA. ${ }^{7}$ Department of Ophthalmology, National University Health Systems, National University of Singapore Singapore 119228, Singapore. ${ }^{8}$ Department of Twin Research and Genetic Epidemiology, King's College London School of Medicine, London SE1 7EH, UK. ${ }^{9}$ School of Optometry and Vision Sciences, Cardiff University, Cardiff CF24 4HQ, UK. ${ }^{10}$ Department of Ophthalmology, University Medical Center Mainz, 55131 Mainz, Germany. ${ }^{11}$ Department of Ophthalmology, Inselspital, University Hospital Bern, CH-3010 Bern, Switzerland. ${ }^{12}$ Medical Research Council Human Genetics Unit, Institute of Genetics and Molecular Medicine, University of Edinburgh, Edinburgh EH4 2XU, Scotland. ${ }^{13}$ Department of Public Health and Primary Care, Institute of Public Health, University of Cambridge School of Clinical Medicine, Cambridge CB2 OSR, UK. ${ }^{14}$ Department of Ophthalmology and Visual Sciences, Kyoto University Graduate School of Medicine, Kyoto 6068507, Japan. ${ }^{15}$ Program in Genetics and Genome Biology, The Hospital for Sick Children and Institute for Medical Sciences, University of Toronto, Toronto Ontario, Canada M5G 1X8. ${ }^{16}$ Department of Clinical Chemistry, Fimlab Laboratories and School of Medicine, University of Tampere, Tampere 33520, Finland. ${ }^{17}$ Statistical Genetics Laboratory, QIMR Berghofer Medical Research Institute, Herston, Brisbane, Queensland 4029, Australia. ${ }^{18}$ Estonian Genome Center, University of Tartu, Tartu 51010, Estonia. ${ }^{19}$ Centre for Eye Research Australia (CERA), Royal Victorian Eye and Ear Hospital, University of Melbourne, Melbourne, Victoria 3002, Australia. 20 Université de Bordeaux, ISPED (Institut de Santé Publique d'Épidémiologie et de Développement), Bordeaux 33000, France. ${ }^{21}$ INSERM, U1219-Bordeaux Population Health Research Center, Bordeaux 33000 , France. 22 Institute of Population Genetics, National Research Council, Sassari 07100, Italy. ${ }^{23}$ Department of Public Health, University of Helsinki, Helsinki 00014, Finland. ${ }^{24}$ Department of Ophthalmology, University of Helsinki and Helsinki University Hospital, Helsinki 00014, Finland. ${ }^{25}$ UCL Institute of Ophthalmology, London SE1 7EH, UK. ${ }^{26}$ Menzies Research Institute Tasmania, University of Tasmania, Hobart, Tasmania 7000, Australia. ${ }^{27}$ Department of Statistical Science, School of Mathematics and Computational Science, Sun Yat-Sen University, Guangzhou 510275, China. ${ }^{28}$ Institute of Medical Biostatistics, Epidemiology and Informatics, University Medical Center Mainz, 55131 Mainz, Germany. ${ }^{29}$ Department of Ophthalmology, King's College London, London SE1 7EH, UK. ${ }^{30}$ Faculty of Medicine, University of Split, Split 21000, Croatia. ${ }^{31}$ Usher Institute for Population Health Sciences and Informatics, University of Edinburgh, Teviot Place, Edinburgh EH8 9AG, Scotland. ${ }^{32}$ Department of Ophthalmology, Sisters of Mercy University Hospital, Zagreb 10000, Croatia. ${ }^{33}$ MRC Integrative Epidemiology Unit (IEU), University of Bristol, Bristol BS8 2BN, UK. ${ }^{34}$ School of Social and Community Medicine, University of Bristol, Bristol BS8 2BN, UK. ${ }^{35}$ Max Planck Institute for Psycholinguistics, Wundtlaan 1, 6525 XD Nijmegen, The Netherlands. ${ }^{36}$ University of Queensland Diamantina Institute, Translational Research Institute, Brisbane, Queensland 4102, Australia. ${ }^{37}$ Department of Genetics, Genomics and Informatics, University of Tennessee Health Science Center, Memphis, Tennessee 38163, USA. ${ }^{38}$ Department of Epidemiology and Biostatistics, Case Western Reserve University, Cleveland, Ohio 44106, USA. ${ }^{39}$ Dardenne Eye Hospital, Bonn-Bad Godesberg, 53177 Bonn, Germany. ${ }^{40}$ Inserm, U1167, Lille 59000, France. ${ }^{41}$ Univ. Lille, U1167, Lille 59000, France. ${ }^{42}$ Université Lille 2, Lille 59000, France. ${ }^{43}$ Research Centre of Applied and Preventive Medicine, University of Turku, Turku 20520, Finland. ${ }^{44}$ Department of Clinical Physiology and Nuclear Medicine, Turku University Hospital, Turku 20520, Finland. ${ }^{45}$ Department of Clinical Physiology, Tampere University Hospital and School of Medicine, University of Tampere, Tampere 33520, Finland. ${ }^{46}$ Clinic for General and Interventional Cardiology, University Heart Center Hamburg, 20246 Hamburg, Germany. ${ }^{47}$ Institute of Human Genetics, Helmholtz Zentrum München, 85764 Neuherberg, Germany. ${ }^{48}$ Institute of Human Genetics, Klinikum rechts der Isar, Technische Universität München, 81675 Munich, Germany. 49 Institute of Genetic Epidemiology, Helmholtz Zentrum München-German Research Center for Environmental Health, 85764 Neuherberg, Germany. ${ }^{50}$ Department of Internal Medicine, Erasmus Medical Center, 3000 CA Rotterdam, The Netherlands. ${ }^{51}$ Netherlands Consortium for Healthy Ageing, Netherlands Genomics Initiative, 2518 AD Hague, The Netherlands. ${ }^{52}$ Beijing Institute of Ophthalmology, Beijing Tongren Hospital, Capital Medical University, Beijing 100044 , China. ${ }^{53}$ Saw Swee Hock School of Public Health, National University of Singapore and National University Health Systems, Singapore 117549, Singapore. 54 National Healthcare Group Eye Institute, Tan Tock Seng Hospital, Singapore 308433, Singapore. ${ }^{55}$ Department of Medicine, National University of Singapore, Singapore 119228, Singapore. ${ }^{56}$ Division of Human Genetics, Genome Institute of Singapore, Singapore 138672, Singapore. ${ }^{57}$ Division of Genetics and Epidemiology, UCL Institute of Ophthalmology, London EC1V 9EL, UK. ${ }^{58}$ NIHR Biomedical Research Centre, Moorfields Eye Hospital NHS Foundation Trust and UCL Institute of Ophthalmology, London EC1V 2PD, UK. ${ }^{59}$ Department of Ophthalmology and Visual Sciences, University of Wisconsin School of Medicine and Public Health, Madison, Wisconsin 53726, USA. ${ }^{60}$ Department of Ophthalmology, Centre for Vision Research, Westmead Institute for Medical Research, University of Sydney, Sydney, New South Wales 2145, Australia. ${ }^{61}$ Department of Ophthalmology and Visual Sciences, School of Medicine and Public Health, University of Wisconsin, Madison, Wisconsin 53705, USA. ${ }^{62}$ State Key Laboratory of Ophthalmology, Zhongshan Ophthalmic Center, Sun YatSen University, Guangzhou 510060, China. ${ }^{63}$ Department of Ophthalmology, Central Hospital of Central Finland, Jyväskylä 40620, Finland. ${ }^{64}$ Gerontology Research Center and Department of Health Sciences, University of Jyväskylä, Jyväskylä 40014, Finland. ${ }^{65}$ Medical Faculty Mannheim, Department of Ophthalmology, Ruprecht-Karls-University Heidelberg, 69115 Mannheim, Germany. ${ }^{66}$ Department of Statistics and Applied Probability, National University of Singapore, Singapore 117546, Singapore. ${ }^{67}$ Centre for Ophthalmology and Visual Science, Lions Eye Institute, University of Western Australia, Perth, Western Australia 6009, Australia. ${ }^{68}$ Department of Ophthalmology, University of Pennsylvania, Philadelphia, Pennsylvania 19104, USA. ${ }^{\star}$ These authors contributed equally to this work. ${ }^{\star \star}$ These authors jointly supervised this work.

$\dagger$ A full list of consortium members appears at the end of the paper

\section{Consortium for Refractive Error and Myopia (CREAM)}

Jugnoo S. Rahi6 $69,70,71$, Jean-François Korobelnik ${ }^{72,73}$, John P. Kemp ${ }^{74}$, Nicholas J. Timpson ${ }^{74}$,

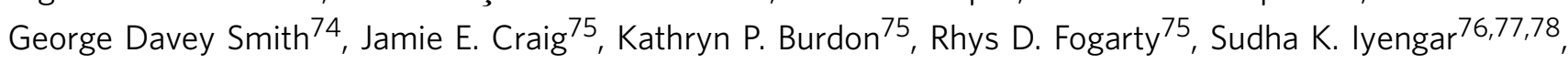
Emily Chew ${ }^{79}$, Sarayut Janmahasatian ${ }^{76}$, Nicholas G. Martin ${ }^{80}$, Stuart MacGregor ${ }^{17}$, Liang Xu ${ }^{52}$, Maria Schache ${ }^{19}$, Vinay Nangia ${ }^{81}$, Songhomitra Panda-Jonas ${ }^{81}$, Alan F. Wright ${ }^{12}$, Jeremy R. Fondran ${ }^{76}$, Jonathan H. Lass ${ }^{76,77}$, Sheng Feng ${ }^{82}$, Jing Hua Zhao ${ }^{83}$, Kay-Tee Khaw ${ }^{13}$, Nick J. Wareham ${ }^{84}$, Taina Rantanen ${ }^{85}$, Jaakko Kaprio $86,87,88$, Chi Pui Pang 89 , Li Jia Chen90, Pancy O. Tam ${ }^{89}$, Vishal Jhanji89,90, Alvin L. Young89,90, Angela Döring ${ }^{91,92}$, Leslie J. Raffel ${ }^{93}$, Mary-Frances Cotch 94 , Xiaohui Li ${ }^{95}$, Shea Ping Yip 96 , Maurice K.H. Yap ${ }^{96}$, Ginevra Biino 97 , Simona Vaccargiu ${ }^{22}$, Maurizio Fossarello ${ }^{22}$, Brian Fleck ${ }^{98}$, Seyhan Yazar ${ }^{19,67}$, 


\section{Jan Willem L. Tideman ${ }^{3,4}$, Milly Tedja 3 ,4, Margaret M. Deangelis ${ }^{99}$, Margaux Morrison ${ }^{100}$, Lindsay Farrer ${ }^{101}$, Xiangtian Zhou ${ }^{102}$, Wei Chen ${ }^{102}$, Nobuhisa Mizuki ${ }^{103}$, Akira Meguro ${ }^{103}$, Kari Matti Mäkelä104}

${ }^{69}$ Medical Research Council Centre of Epidemiology for Child Health, Institute of Child Health, University College London, London WC1E 6BT, UK; ${ }^{70}$ Institute of Ophthalmology, Moorfields Eye Hospital, London EC1V 2PD, UK; ${ }^{71}$ Ulverscroft Vision Research Group, University College London, London WC1E 6BT, UK;

72Université de Bordeaux, 33400 Talence, France; ${ }^{73}$ INSERM (Institut National de la Santé Et de la Recherche Médicale), ISPED (Institut de Santé Publique d'Épidémiologie et de Développement), Centre INSERM U897-Epidemiologie-Biostatistique, 33076 Bordeaux, France; ${ }^{74}$ MRC Integrative Epidemiology Unit (IEU), The University of Bristol, Bristol BS8 2BN, UK; ${ }^{75}$ Department of Ophthalmology, Flinders University, Adelaide, South Australia 5001, Australia;

${ }^{76}$ Department of Epidemiology and Biostatistics, Case Western Reserve University, Cleveland, Ohio 44106, USA; ${ }^{77}$ Department of Ophthalmology and Visual Sciences, Case Western Reserve University and University Hospitals Eye Institute, Cleveland, Ohio 44106, USA; ${ }^{78}$ Department of Genetics, Case Western Reserve University, Cleveland, Ohio 44106, USA; ${ }^{79}$ National Eye Institute, National Institutes of Health, Bethesda, Maryland 20892, USA; ${ }^{80}$ Genetic Epidemiology Laboratory, QIMR Berghofer Medical Research Institute, Herston, Brisbane, Queensland 4029, Australia; ${ }^{81}$ Suraj Eye Institute, Nagpur, Maharashtra 440001, India; ${ }^{82}$ Department of Pediatric Ophthalmology, Duke Eye Center For Human Genetics, Durham, North Carolina 27710, USA; ${ }^{83}$ MRC Epidemiology Unit, Institute of Metabolic Sciences, University of Cambridge, Cambridge CB2 1TN, UK; ${ }^{84}$ MRC Epidemiology Unit, Institute of Metabolic Science, Addenbrooke's Hospital, Cambridge CB2 OQQ, UK; ${ }^{85}$ Gerontology Research Center, University of Jyväskylä, Jyväskylä 40014, Finland; ${ }^{86}$ Department of Public Health, University of Helsinki, Helsinki 00014, Finland; ${ }^{87}$ Institute for Molecular Medicine, University of Helsinki, Helsinki 00014 , Finland; ${ }^{88}$ Department of Mental Health and Alcohol Abuse Services, National Institute for Health and Welfare, Helsinki 00271 , Finland; ${ }^{89}$ Department of Ophthalmology and Visual Sciences, Hong Kong Eye Hospital, The Chinese University of Hong Kong, Kowloon, Hong Kong; ${ }^{90}$ Department of Ophthalmology and Visual Sciences, Prince of Wales Hospital, The Chinese University of Hong Kong, Shatin, Hong Kong; ${ }^{91}$ Institute of Epidemiology I, Helmholtz Zentrum München, German Research Center for Environmental Health, 85764 Neuherberg, Germany; ${ }^{92}$ Institute of Epidemiology II, Helmholtz Zentrum München, German Research Center for Environmental Health, 85764 Neuherberg, Germany; ${ }^{93}$ Medical Genetics Institute, Cedars-Sinai Medical Center, Los Angeles, California 90048, USA; ${ }^{94}$ Division of Epidemiology and Clinical Applications, National Eye Institute, Bethesda, Maryland 20892, USA; ${ }^{95}$ Institute for Translational Genomics and Population Sciences, Los Angeles Biomedical Research Institute, Harbor-UCLA Medical Center, Los Angeles, California 90502, USA; ${ }^{96}$ Department of Health Technology and Informatics, The Hong Kong Polytechnic University, Hong Kong, Hong Kong; ${ }^{97}$ Institute of Molecular Genetics, National Research Council, Pavia 27100, Italy; ${ }^{98}$ Princess Alexandra Eye Pavilion, Edinburgh EH3 9HA, UK; ${ }^{99}$ Department of Ophthalmology and Visual Sciences, Moran Eye Center, University of Utah, Salt Lake City, Utah 84132, USA; ${ }^{100}$ Department of Ophthalmology and Visual Sciences, John Moran Eye Center, University of Utah, Salt Lake City, Utah 84132, USA; ${ }^{101}$ Departments of Medicine (Biomedical Genetics), Ophthalmology, Neurology, Epidemiology and Biostatistics, Boston University Schools of Medicine and Public Health, Boston, Massachusetts 02118, USA; 102School of ophthalmology and optometry, Wenzhou Medical University, Wenzhou 325035, China; ${ }^{103}$ Department of Ophthalmology, Yokohama City University School of Medicine, Yokohama, Kanagawa 236-0027, Japan; ${ }^{104}$ Department of Clinical Chemistry, Fimlab Laboratories and School of Medicine, University of Tampere, Tampere 33014, Finland. 